\title{
Hypersensitivity reaction to dapsone. Four case reports
}

\author{
MARY S JOSEPH \\ Nou Sombou Leprosy Hospital, Khon Kaen, Thailand
}

Accepted for publication 10 June 1985

Summary Hypersensitivity reaction to the most commonly used antileprosy drug, dapsone, is becoming an increasingly important problem in the field of leprosy treatment. This undesired reaction to dapsone is more common than was thought previously. In this article 4 cases seen at the Nonsombun Leprosy Hospital during the years 1982-83 are reported. The 4 leprosy patients (2 cases of BL, I case of BT and 1 case of TT) presented with cutaneous and systemic manifestations of hypersensitivity to dapsone. The diagnosis was confirmed by trial dose in 3 cases.

\section{Introduction}

Dapsone is still the drug of choice in treating leprosy in most countries. It is also being used in many other skin diseases. We have the impression that we are seeing hypersensitivity and other side effects to this drug more commonly than before.

Hypersensitivity is confined to the first 6 weeks of treatment. The skin manifestations of hypersensitivity reaction to dapsone are exanthematous eruptions, exfoliative dermatitis, toxic epidermal necrolysis and Stevens-Johnson syndrome (erythema multiforme bullosum). The systemic manifestations include fever, eosinophilia, mononucleosis, lymphadenopathy, hepatitis, etc. A fatal hypersensitivity reaction known as 'DDS Syndrome' was described by leprologists in the early years of its use.

\section{Case Reports}

No. 1. A 50-year-old female patient (weight $35-40 \mathrm{~kg}$ ), with active BL leprosy, was registered in the OPD clinic in 1982 and was started on antileprosy drugs including dapsone (50 mg daily) clofazimine and rifampicin (according to the national regime). She came back in the fourth week with reddish itchy papular rashes which were more marked on the sun exposed areas, namely, the face, neck, 
upper chest and forearms. There were rashes on the trunk too. The patient complained of fever and weakness and there was associated redness of eyes and generalized lymphadenopathy. A few blisters and small ulcers were seen on the lower lip. The original leprosy lesions were obscured due to the rash.

She was admitted and all drugs were stopped temporarily. Only antihistamines and topical lotions were given. After 2 weeks when the skin was clean she was given a trial dose of dapsone $50 \mathrm{mg}$ and kept under close clinical observation. Within 4 hours she complained of feeling 'hot' and developed itching and burning associated with erythematous papular rashes mainly on the face and upper extremities. The conjunctivae were congested too. All symptoms subsided following antihistamines. Now she is on alternative treatment for leprosy.

No. 2. A 24-year-old female patient (weight $52 \mathrm{~kg}$ ) with active BL leprosy was started on treatment with all 3 drugs in the OPD clinic in 1983. After about a month she was brought back with jaundice and skin rashes. On examination the patient was quite weak, febrile and moderately jaundiced. There was generalized lymphadenopathy and conjunctivitis. The liver was enlarged and tender. The skin rashes were miliariform and mainly over the face, trunk and upper extremities, and associated with itching and burning. The lips were blistered. The leprosy lesions were not visible except the few papules and nodules.

The patient was admitted, all antileprosy drugs were stopped and she was treated with prednisolone and topical lotions only. After about 2 months she was fit for discharge and was sent out on clofazimine. She was not challenged with dapsone since it was thought very risky, considering the severity of her initial illness.

During the course in the hospital and while still on small doses of prednisolone, she had developed repeated rashes with itching mainly on her face and forearms whenever she went out in the sun.

No. 3. A 35-year-old male patient (weight $-60 \mathrm{~kg}$ ) presented at the OPD clinic in 1983 with generalized erythroderma and exfoliative dermatitis. On further questioning he showed a packet of dapsone $100 \mathrm{mg}$ tablets which he had received from a local health centre and had been taking for over one month. $\mathrm{He}$ had also brought supplies of antihistamines as well as prednisolone tablets which had been given by 3 different private doctors over the past 3 weeks. But none of them had instructed him to stop dapsone-probably he did not volunteer to tell them that he was taking dapsone. It was stopped on admission here.

The patient complained of itching and burning all over the body, as well as fever and weakness. On examination he had generalized lymphadenopathy. The leprosy patches were not clearly visible but there were fairly large islands of normal looking skin on both legs. The patient claimed that those were the sites of hypopigmented patches earlier, for which he was given dapsone by the health centre. The islands were dry and anaesthetic, not itchy and there was no redness or exfoliation on them. One lateral poplitial nerve was thickened. He was clinically classified as BT leprosy. 
The dermatitis cleared in 6 weeks on treatment with prednisolone and topical applications. He too developed mild rash on face and forearms when exposed to the sun after prednisolone was stopped, but required only antihistamines. Later when all lesions were cleared he was given a trial dose of dapsone $50 \mathrm{mg}$. After about 2 hours he complained of feeling feverish and burning over the face. This was soon followed by erythema, itching and papular rashes over face, trunk and upper extremities. The face was slightly puffy and the eyes were congested. All symptoms subsided with antihistamines. He was discharged after another week with no specific treatment, but with the instruction to return after 2 months for reexamination for activity of leprosy and alternative treatment if needed. But he never returned.

No. 4. A 45-year-old female patient (weight $59 \mathrm{~kg}$ ) was brought to the OPD in 1983 with severe exfoliative dermatitis following 3 weeks of dapsone $100 \mathrm{mg}$ daily, given at the local health centre. The face was oedematous and the lips were blistered and ulcerated. There were ulcers even on the oral mucosa. The patient was febrile and had generalized lymphadenopathy and conjunctivitis.

The patient was admitted, dapsone was stopped and the dermatitis was treated with prednisolone and topical applications. After about 6 weeks when all lesions had cleared and when she was off prednisolone she was given a trial dose of dapsone $50 \mathrm{mg}$ under close clinical observation. Within 3-4 hours she developed symptoms such as fever, itching and burning over the face with exanthematous rashes on the face, trunk and upper extremities. Symptoms subsided with antihistamines. Later she was examined for leprosy. There was only a small area of anaesthesia on the right elbow, with no nerve thickening. The patient said that

Table 1. Summary of the main manifestations in the 4 patients
Cases
Cutaneous manifestations
Systemic manifestations

No. 1

F $50 \mathrm{yr}$

$\mathrm{BL}$

No. 2

F 24 yr

BL

\section{No. 3}

M $35 \mathrm{yr}$

BT

No. 4

F 45 yr

TT
Itching, burning, exanthematous rash

Fever, weakness, lymphadenopathy, congestion of eyes, ulcers and blisters on lip

Fever, weakness, conjunctivitis, hepatitis, lymphadenopathy

Fever, weakness, lymphadenopathy

Itching, burning, erythroderma, exfoliation, recurrence of rash on exposure to sun, leprosy lesions free of involvement

Itching, burning, exfoliative dermatitis
Fever, weakness, swelling of face, lymphadenopathy, conjunctivitis, ulcers of mouth 
she had a patch over the same site. So she was classified as a case of TT leprosy, the activity of which was to be decided later. She was not given any treatment on discharge, but was instructed to come back after 2 months for re-assessment. She did not return in 1983, but presented again in July, 1984 with severe exfoliative dermatitis and a packet of dapsone $50 \mathrm{mg}$ tablets! The tablets were forced on her by the local health worker when he found out that she was not taking dapsone. She admitted taking just a single tablet, after which she developed the dermatitis and reported here.

In all these 4 patients dapsone hypersensitivity was clinically diagnosed and this was supported by the fact that symptoms subsided when dapsone was stopped and all 4 were treated with prednisolone/antihistamines. In the third patient, though he was treated with prednisolone and antihistamines along with dapsone, his symptoms and signs did not improve until the offending drug, dapsone, was stopped. In all but one patient the diagnosis was confirmed by reproducing the signs and symptoms when a single trial dose was given. No significant mental disturbance occurred in any of the 4 patients. Summary of the main manifestations in the 4 patients is shown in Table 1.

\section{Discussion}

The cutaneous and systemic manifestations presented by all 4 patients were compatible with those of dapsone hypersensitivity ${ }^{10}$ as well as the DADPS Syndrome described earlier. ${ }^{2}$ All patients fulfilled most of the criteria for hypersensitivity reaction, namely the symptoms occurred within 6 weeks of starting dapsone, and the manifestations included most of the following: fever, lymphadenopathy, hepatitis and exanthematous skin rash. ${ }^{4}$ Two patients had severe skin manifestation in the form of exfoliative dermatitis. Due to lack of laboratory facilities a complete blood count was not done to look for eosinophilia or mononucleosis. The sequence of syndrome usually is dermatitis, hepatitis, lymphadenopathy and mononucleosis of which dermatitis is always present though the others may or may not be present. ${ }^{2}$ Such cases may even prove fatal if not diagnosed and treated in time and/or manifest in the form of more severe dermatological emergencies such as toxic epidermal necrolysis ${ }^{5}$ and StevensJohnson syndrome (erythema multiforme bullosum). ${ }^{1}$

Such cutaneous and systemic complications of dapsone in the field of leprosy had been encountered by leprologists even earlier. ${ }^{7,8}$ The author had come across similar cases while a medical officer at the Schieffelin Leprosy Research and Training Centre, Karigiri, India. ${ }^{9}$ But in some of those cases the manifestations were more of an allergic nature (symptoms appearing even after a single dose) rather than hypersensitivity.

Hypersensitivity is not dose related ${ }^{10}$ as in the case of toxicity and therefore giving the drug in gradually increasing small doses may not be of help. ${ }^{3}$ In a 
previously sensitized person even a small dose may be dangerous. With the availability of alternative and effective antileprosy drugs, it is not necessary to desensitize these patients presently. Moreover, this practice may predispose to dapsone resistance. But it should be remembered that such patients with proved hypersensitivity should be given alternative treatment as severe hypersensitivity can be fatal.

One of the purposes of this paper is to point out that the BT leprosy lesions were not involved in the hypersensitivity manifestation. The lesions were free of symptoms or signs. A similar feature was noticed by the author in the patients in India, but to the author's knowledge such a feature has not been documented in earlier reports. The possible explanation is that in BT (and TT) lesions due to the complete damage of the sympathetic nerve fibres, there is absence of axon reflex which is responsible for the histamine response to the offending allergen, leading to the cutaneous manifestations seen. This fact is supported by the finding of no such feature in BL lesions where there is no complete damage to the nerve fibres in early stages.

The reason for 2 of the patients developing fresh skin rashes on exposure to sun when no fresh dose of the drug was taken could be that in highly sensitized subjects even small doses of the offending drug, when combined with ultraviolet light are sufficient to trigger the hypersensitive reaction. It is reasonable to assume that in previously involved and damaged skin there may be prolonged retention of small amounts of the photoallergic bacteriostatic agent.

It is important to remember that hypersensitivity reactions may not be as uncommon as was previously thought. ${ }^{6}$ Dapsone is usually given under field conditions by paramedical workers and patients who receive dapsone alone do not get such close supervision as those on rifampicin or lamprene. Such patients, when they develop dermatitis, hepatitis, etc, are usually referred to the general hospitals and thus may not even come to the notice of leprologists. So the actual number of such cases could be even more than those so far documented.

\section{Acknowledgments}

I am grateful to all the above patients who have been very co-operative. Thanks are due to the director of the leprosarium for granting me permission to publish. Thanks are also due to the authorities of the doctors' library at Khon Kaen University Medical School for permitting me to use their materials for reference.

\section{References}

' Dutta RK. Erythema Multiforme Bullosum due to Dapsone. Lepr India, 1980; 52 (2) 306-9.

2 Allday J, Barnes J. Toxic effects of Diamino Diphenylsulphone in treatment of leprosy. Lancet, 1951; 2: 205-6. 
Jelliffe DB. Toxic hepatitis caused by Diamino Diphenylsulphone. L.ancet, 195I; 1: 1343

4 Jopling WH. Side effects of antileprosy drugs in common use. Editorial, Le'pr Rer, 1983; 54: 26170 .

${ }^{5}$ Katoch K, Ramu G, Usha Ramanathan. Toxic Epidermal Necrolysis (Lyell Syndrome). A case report. Lepr India, 1983; 55 (1).

6 Lancet, 1981, 2: 184 5. Adverse Reactions to Dapsone.

7 Ramanujam K, Ramu G. Toxic reactions to parent sulphone. Report of four cases. Leper Indica, 1968; 40 (1) 1-7.

${ }^{8}$ Sardari Lal, Garg BR. Sulphone induced exfoliative dermatitis and hepatitis. Lepr India, 1980; 52 (2) $302-5$.

${ }^{9}$ Scholastica M, Arunthathi S, Taylor PM. Dapsone Hypersensitivity Reaction. Unpublished paper, presented at the XII Biennial Conference of the Indian Association of Leprologists, at Agra, India, 7-12 Sep. 1981.

10 Tomecki KJ, Catalano CJ. Dapsone Hypersensitivity. Archives of Dermatology, 1981; 117: 38-9. 